\title{
IM SCHATTEN DES KAISERS: \\ ÜBERLEGUNGEN ZU L. MINICIUS NATALIS QUADRONIUS VERUS UND SEINER BEZIEHUNG ZU HADRIAN ${ }^{1}$ \\ Von \\ ANDREAS KRIECKHAUS
}

Für Anthony R. Birley

zum 08.10.2002

I

Der Hippodrom von Olympia im August $129:^{2}$ Die Quadriga des römischen Senators L. Minicius Natalis Quadronius Verus (der „Jüngere“), Sohn des Suffektkonsuls von 106, L. Minicius Natalis (der „Ältere“) aus Barcino, siegt bei den olympischen Spielen im Wagenrennen, einer elementaren Disziplin der hippischen Agone. $^{3}$ Sie wird vermutlich von einem professionellen Athleten gelenkt, während der $\sigma \tau \rho \alpha \tau \eta \gamma \imath$ ós (praetorius) Minicius Natalis - noch ohne ein neues $\mathrm{Amt}^{4}$ - als Besitzer des Gespannes

\footnotetext{
${ }^{1}$ Einige häufige bzw. besondere Abkürzungen:

Birley 1997 = A.R. Birley, Hadrian. The Restless Emperor (London-New York 1997)

Caballos Rufino $1990=\mathrm{A}$. Caballos Rufino, Los senadores hispanorromanos y la romanizacion de Hispania (siglos I al III p.C.), 2 Bände (Ecija 1990)

Groag 1932 = E. Groag, 'Minicius', $R E$ XV (Stuttgart 1932), 1828ff., Nr. 18 u. 19

Habicht 1985 = C. Habicht, Pausanias und seine „Beschreibung Griechenlands “(München 1985)

IRB = S. Mariner Bigorra (Hg.), Inscripciones Romanas de Barcelona (Barcelona 1973)

IRC IV = G. Fabre, M. Mayer u. I. Rodà (Hgg.), Inscriptions Romaines de Catalogne, Band IV (Paris 1997)

Roman Papers I-VII = E. Badian u. A.R. Birley (Hgg.), Ronald Syme, Roman Papers, 7 Bände (Oxford 1979-1991)

Verrié 1989 = F.-P. Verrié, 'La inscripció olímpica del barcinonense Lucius Minicius Natalis Quadronius Verus', in: Espacio, tiempo y forma (arqueol.), Revista de la Facultad de Geografia e Historia 2 (1989), 303ff.

${ }^{2}$ Der Hippodrom von Olympia ist lokalisiert, jedoch noch nicht ausgegraben. Eine Rekonstruktion legt J. Ebert, 'Neues zum Hippodrom und zu den hippischen Konkurrenzen in Olympia', Nikephoros 2 (1989), 89ff. vor.

${ }^{3}$ Zu L. Minicius Natalis Quadronius Verus vgl. PIR ${ }^{2}$ M 620; Groag 1932, 1836ff.; W. Eck, 'Minicius', RE Suppl. XIV (Stuttgart 1974), 283; R. Etienne, ,Les sénateurs espagnols sous Trajan et Hadrien', in Les Empereurs romains d'Espagne (Paris 1965), 72; Caballos Rufino 1990 I,1, 227ff., Nr. 128. Zu L. Minicius Natalis vgl. PIR ${ }^{2}$ M 619; Groag 1932, 1828ff.; Etienne 1965, a.a.O., 62f.; Caballos Rufino 1990 I, 1, 226f., Nr. 127.

${ }^{4}$ L. Minicius Natalis Quadronius Verus sollte erst um das Jahr 130 als Legionslegat der legio VI Victrix in der Provinz Britannia, deren Statthalter zum damaligen Zeitpunkt Sex. Iulius Severus war, wieder in Erscheinung treten. Eben dieser Iulius Severus hatte vor 120 die legio XIV Gemina kommandiert, in der der jüngere Minicius Natalis während dieser Zeit als Militärtribun tätig gewesen war. Somit ist es nicht unwahrscheinlich, daß Severus um 130 Kaiser Hadrian seinen ehemaligen Tribun für den Posten des Legionslegaten empfahl. Vgl. dazu A.R. Birley, The Fasti of Roman Britain
} 
auf der Tribüne sitzt und nach dem Sieg seines Schützlings den Ölkranz des Siegers in der Vorhalle des Zeus Olympios-Tempels entgegennimmt. ${ }^{5}$

So etwa könnte man sich die Szenerie vorstellen, die sich bei der 227. Olympiade darbot. Eine Beschreibung der Ereignisse um das Wagenrennen fehlt; daß die Quadriga des jüngeren Minicius Natalis den Sieg errang, wissen wir lediglich durch eine Inschrift aus Olympia, von der deutsche Archäologen zu Beginn des Jahres 1878 zwei Fragmente entdeckten. Diese wurden durch Karl Purgold und Wilhelm Dittenberger ediert. ${ }^{6}$ Ende der achtziger Jahre des letzten Jahrhunderts kam ein drittes Fragment hinzu, so daß der zweizeilige Text nun nahezu vollständig vorliegt:

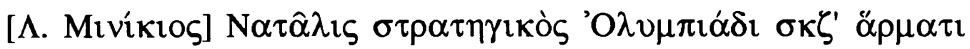

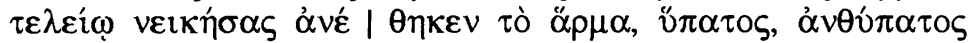

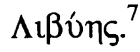

Die Art und Beschaffenheit des Inschriftenträgers - drei Kalksteinblöcke, insgesamt ca. 2,8 m breit, mit diversen Vertiefungen auf der Oberseite -

(Oxford 1981), 108 und 245f. sowie Anm. 50.

${ }^{5} \mathrm{Da}$ der Pferdesport in der Antike ein Sport der Reichen war und man grundsätzlich zwischen dem Besitzer respektive Geldgeber und dem Wagenlenker zu unterscheiden hat (auch bei der Siegerehrung, bei der grundsätzlich der Rennstallbesitzer den Kranz erhielt), braucht wohl nicht weiter ausgeführt zu werden. Für den jüngeren Minicius Natalis betont I. Rodà dies dennoch nochmals: 'Luci Minici Natal Quadroni Ver i la societat barcelonina del seu temps', Revista de Catalunya 32 (1988), 46f. Vgl. allgemein zur Geschichte von Olympia, den Wettkämpfen und Bauten H.-V. Herrmann, Olympia. Heiligtum und Wettkampfstätte (München 1972) und A. Mallwitz, Olympia und seine Bauten (Darmstadt 1972). Den Ablauf der Ereignisse bei den olympischen Siegesfeiern und die Aufstellung der Siegerstatuen beschreibt H. Buhmann, Der Sieg in Olympia und in den anderen panhellenischen Spielen (München ${ }^{2} 1975$ ), $53 \mathrm{ff}$.

${ }^{6}$ IOlymp. $236=$ Sylloge 840 . Zwei spanische Beiträge haben sich in jüngerer Vergangenheit intensiver mit der Inschrift auseinandergesetzt: Verrié 1989, 303ff. und J.-V. Rodríguez Adrados, 'El barcelonés Lucio Minicio Nadal, campeón olímpico', in: J. Zaragoza \& A. González Senmartí (Hgg.), Homenatge a Josep Alsina. Actes del Xè simposi de la secció catalana de la SEEC I (Tarragona 1992), $431 \mathrm{ff}$. In beiden Fällen - insbesondere jedoch im zweiten - ist kritisch anzumerken, daß wichtige außerspanische Forschungsliteratur nicht verwendet wurde. Rodríguez Adrados kennt zudem offenbar den drei Jahre zuvor erschienenen Beitrag seines Landsmannes Verrié nicht, da er ihn nicht zitiert. Ein Blick in SEG 35 (1985), 385. 1775 und SEG 42 (1992), 388 hätte Abhilfe schaffen können. Hinzu kommt, daß Rodriguez Adrados die völlig abwegige These vertritt, der ältere Minicius Natalis sei der Olympiasieger von 129 gewesen. Dies macht er vor allem an seiner m.E. höchst problematischen Deutung des Begriffes $\sigma \tau p \alpha \tau \eta \gamma$ ikós als „General“ und nicht als praetorius fest.

${ }^{7}$ Vgl. zum dritten Fragment Verrié 1989, 305f. Die von ihm vorgeschlagene Ergänzung $\Lambda$. Mivíkı

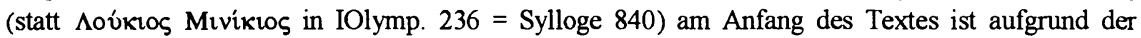
Textsymmetrie in beiden Zeilen nach der Entdeckung des dritten Fragments nun mehr als wahrscheinlich. Verrié zitiert keine Erstpublikation des neuen Fragments; außer in seinem Beitrag, der in SEG 42 (1992), 388 Erwähnung findet, ist es m.W. bisher nicht veröffentlicht worden. 
weist darauf hin, daß es sich ursprünglich um ein Monument mit Wagen und Wagenlenker handelte, somit also um das Siegesdenkmal des jüngeren Minicius Natalis. ${ }^{8}$ Aufgrund der aktuellen Fundsituation muß man von einer Gesamtbreite von ca. 3,5 m und vier Quadern ausgehen. ${ }^{9}$ Entdeckt wurden die Steine in der im Westen von Olympia gelegenen Palaestra, also ein wenig außerhalb der Altis, des Kultplatzes, wo sich noch zu Pausanias' Zeit zahlreiche Statuen, deren Aufstellung den Athleten bzw. Mäzenen nach deren Siegen erlaubt wurde, um den Zeustempel gruppierten. ${ }^{10}$

Betrachtet man den Inschriftentext, so drängt sich eine Frage auf, die auch den Ablauf der Ereignisse nach dem Wagenrennen betriff: Ließ der jüngere Minicius Natalis nach seinem Olympiasieg das ihm zugebilligte Monument schon 129 errichten, oder erst (frühestens) um 154? Die Tatsache, daß der Senator zum Zeitpunkt des Sieges Prätorier war, scheint zunächst für die erste Variante zu sprechen, doch müßte man dann die am Ende des Textes aufgeführten Ämter Suffektkonsulat (139) und Prokonsulat von Africa proconsularis (um 154) als spätere Ergänzungen ansehen, wie dies in der Vergangenheit auch zum Teil geschehen ist. ${ }^{11}$ Wesentlich

\footnotetext{
${ }^{8} \mathrm{Zu}$ den olympischen Siegerstatuen vgl. grundlegend H.-V. Herrmann, 'Die Siegerstatuen von Olympia', Nikephoros 1 (1988), 119ff., der am Ende seines Beitrages eine ausführliche Liste aller belegten Siegerstatuen bietet. Vgl. ferner G. Lippold, 'Siegerstatuen', RE II A (Stuttgart 1923), 2265ff. Die maßgebliche Sammlung aller bekannten Olympiasieger der Antike stellt immer noch L. Moretti, Olympionikai. I vincitori negli antichi agoni olimpici (Rom 1957) dar, jetzt ergänzt durch ein Supplement desselben Autors: 'Nuovo supplemento al catalogo degli Olympionikai', Miscellanea Greca e Romana 12 (1987), 67ff. Allerdings findet sich in diesem Supplement keine neue Literatur zu Minicius Natalis.

${ }^{9}$ Für Einzelheiten bezüglich der Beschaffenheit und Maße vgl. IOlymp. 236 und Verrié 1989, 305ff. Verrié macht in seinem Beitrag deutlich, daß das Monument ursprünglich aus vier Kalksteinblöcken bestand. Der fehlende Block konnte bei den bisherigen Grabungen noch nicht gefunden werden. Diesen Hinweis verdanke ich Prof. Dr. U. Sinn (Würzburg).

${ }^{10} \mathrm{Vgl}$. zur Entdeckung der Steine IOlymp. 236 und Verrié 1989, 305. Zumindest die 1878 gefundenen Fragmente a und $\mathrm{b}$ waren als Spolien in der byzantinischen Aufmauerung der Ostmauer der Palaestra wiederverwendet worden. Pausanias schildert im 6. Buch seiner „Beschreibung Griechenlands“ (Pausanias, Graeciae descriptio 6.1-18) bei seiner Siegerstatuenperiegese, welche Monumente er Mitte des 2. Jahrhunderts in der Altis von Olympia vorfand.

${ }^{11}$ Beispielsweise durch Dittenberger in Sylloge 840 (Consulatus et proconsulatus postea additi videntur), Groag 1932, 1842 (,Die beiden höchsten Ämter sind [...] wohl erst nachträglich

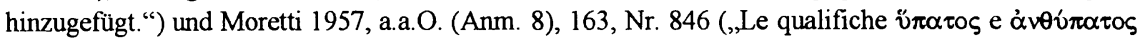
nell'iscrizione di Olimpia sembrano aggiunte in un secondo tiempo perché il vincitore si qualifica

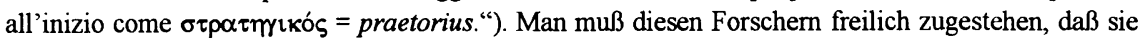
die heute gültige Datierung von Suffektkonsulat und Prokonsulat des jüngeren Minicius Natalis noch nicht kannten (geschweige denn das neugefundene dritte Fragment der Inschrift), somit also von falschen Voraussetzungen ausgingen. Zur Statthalterschaft vgl. B.E. Thomasson, Fasti Africani. Senatorische und ritterliche Amtsträger in den römischen Provinzen Nordafrikas von Augustus bis Diokletian (Stockholm 1996), 61f., Nr. 74.
} 
überzeugender hingegen mutet die zweite Variante an: Zieht man mit Christian Habicht $\sigma \tau \rho \alpha \tau \eta \gamma$ кó

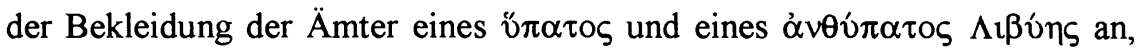
so wird durch die beiden Verben eine Trennung von Sieg und Weihung deutlich; der Sieg fand 129 statt, die Weihung etwa 25 Jahre später. ${ }^{12}$ Diese Trennung war keineswegs ungewöhnlich, wie der Reiseschriftsteller Pausanias, der Olympia aus eigener Anschauung kannte, mehrmals in seiner "Beschreibung Griechenlands“ andeutet. ${ }^{13}$

Hier ist ein Hinweis von Wilhelm Gurlitt beachtenswert, der bereits 1890 bemerkte, daß man die Inschrift mit einer Textpassage bei Pausanias in Verbindung bringen dürfe, wo dieser einen römischen Senator erwähnt, der „zu seiner (sc. Pausanias") Zeit" nach einem Sieg im Wagenrennen in Olympia eine Bronzestatue mit dazugehöriger Inschrift als Siegesdenkmal errichten lassen wollte und bei den Grabungen in unmittelbarer Nähe der Oinomaossäule auf Waffen, Zaumzeug und Gebisse stieß. ${ }^{14}$ Gegen Gurlitts heutzutage weitgehend akzeptierte These $^{15}$ wurden in der Vergangenheit gewisse Bedenken angemeldet: Einerseits berief man sich auf das bereits oben erwähnte - wie wir heute wissen - falsche zeitliche Argument, ${ }^{16}$ andererseits wurde darauf hingewiesen, daß sich die bei Pausanias beschriebene Statue nicht mit dem Wagendenkmal des Minicius Natalis in Einklang bringen lasse. ${ }^{17}$ Es muß jedoch zweierlei angemerkt werden: Zum einen kam die Errichtung zweier unterschiedlicher Monumente durch einen Olympiasieger aus den oben genannten Gründen der Trennung von Sieg und

\footnotetext{
${ }^{12}$ So Habicht 1985, 183f. Auch Verrié 1989, 307ff. vertritt die These einer späteren Weihung, allerdings ganz offensichtlich ohne Kenntnis des Buches von Habicht und dessen philologischer Argumentation. Er stützt sich bei seinen Ausführungen vor allem auf materielle und technische Aspekte in bezug auf die Beschaffenheit der drei vorhandenen Quader bzw. der Inschrift darauf und spricht sich vehement gegen eine spätere Ergänzung von Konsulat und Prokonsulat aus.

${ }^{13}$ Pausanias, Graeciae descriptio 6.10.4, 6.10.6 und 6.16,6. Vgl. dazu Habicht 1985, 183.

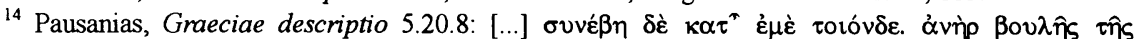

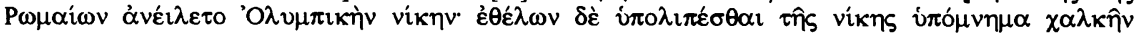

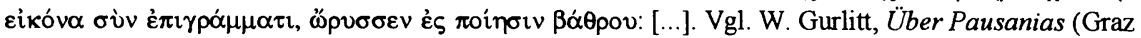
1890), 421, Anm. 37.

${ }^{15}$ Vgl. etwa Birley 1997, 216 und 343; Verrié 1989, 307ff., Anm. 7; Habicht 1985, 183ff.; Rodríguez Adrados 1992, a.a.O. (Anm. 6), 431f. H.-V. Herrmann (Herrmann 1988, a.a.O. [Anm. 8], 182) listet das Monument des Minicius Natalis unter „Siegerstatuen, die nicht bei Pausanias erwähnt, aber archäologisch-epigraphisch greifbar sind" auf, was darauf schließen läßt, daß dem Autor entweder die Diskussion über Pausanias, Graeciae descriptio 5.20.8 unbekannt ist oder er der These nicht folgt.

${ }^{16} \mathrm{Zu}$ den Befürwortern dieser These vgl. Anm. 11. Zudem zeigt C. Habicht (Habicht 1985, 181ff.), daß die Pausaniasstelle (vgl. Anm. 14) zeitlich sehr wohl in die zwanziger Jahre des 2. Jahrhunderts eingeordnet werden kann, indem er den Ausdruck $\kappa \alpha \tau^{\uparrow} \dot{\varepsilon} \mu \dot{\varepsilon}$ dementsprechend deutet.

${ }^{17}$ Bedenken bezüglich der Beschaffenheit des Denkmals äußern etwa Purgold in IOlymp. 236 und Groag 1932, 1842.
} 
Weihung durchaus vor, ${ }^{18}$ zum anderen ist es möglich, daß Pausanias, dessen Augenmerk keineswegs auf römische Olympiasieger gerichtet war, „sich sehr wohl mit einer etwas großzügigen Bezeichnung der Form eines Denkmals begnügt haben könne, das für ihn ohne Interesse war. “19 Dies würde bedeuten, daß er mit der „Bronzestatue“ doch das minicische Wagendenkmal meinte.

Aus den vorausgegangenen Bemerkungen ließe sich nun hypothetisch folgender Ablauf der Ereignisse rekonstruieren: Der Prätorier L. Minicius Natalis Quadronius Verus verzichtete nach dem Sieg seiner Quadriga im Sommer 129 aus unbekannten Gründen auf eine sofortige Statuenweihung. Viele Jahre später, nach seinem Engagement in Africa proconsularis um 154, hat er dann, mittlerweile mit einer weitaus größeren auctoritas und dignitas ausgestattet, in der Nähe der Oinomaossäule inmitten der Altis ein Monument mit Wagen und Wagenlenker errichten lassen, um so an seinen Sieg zu erinnern und den Akt der Weihung zu vollenden. ${ }^{20}$ Pausanias berichtet von sich, er habe den Ausschachtungsarbeiten beigewohnt. ${ }^{21}$ Dieses Ereignis trug sich zu einer Zeit zu, als Olympia gerade die 233. Olympiade erlebt hatte (153) und quasi zeitgleich die bedeutende Stiftung einer Exedra mit dazugehörigem Aquädukt durch den mit Hadrian

${ }^{18}$ Zur möglichen Aufstellung zweier Monumente vgl. Habicht 1985, 184, der u.a. auf Pausanias, Graeciae descriptio 6.10.6 verweist. Vgl. auch Anm. 12 zur Trennung von Sieg und Weihung bei Minicius Natalis.

${ }^{19}$ Habicht 1985, 184. So auch E. Groag (Groag 1932, 1842), der bemerkt: „Indes besteht die Möglichkeit, daß der Perieget keinen Wert darauf gelegt hat, die Beschaffenheit des Denkmals, das für ihn ohne jedes Interesse war, genauer zu bezeichnen." Pausanias' Werk ist geprägt von dessen Vorliebe für die archaische und klassische griechische Zeit. Hellenismus und Römerzeit kommen dagegen sehr kurz. Man sollte jedoch nicht pauschalisieren und behaupten, Pausanias habe die Römer an sich als Feinde betrachtet, sondern seine zweifellos vorhandenen Ressentiments auf die Herrschaft der Römer in Griechenland beziehen. Diese gemäßigte Ansicht vertraten zuletzt K.W. Arafat, Pausanias' Greece. Ancient Artists and Roman Rulers (Cambridge 1996), bes. 202ff. und Habicht 1985, $118 \mathrm{ff}$. Pausanias' völliger Verzicht auf die Erwähnung römischer Siegerstatuen und -namen bei seinem literarischem Rundgang durch die Altis (Pausanias, Graeciae descriptio 6.1-18) dürfte also primär mit den oben geschilderten, von ihm in seinem Werk gesetzten Prioritäten zusammenhängen. Vgl. Herrmann 1988, a.a.O. (Anm. 8), 123ff., der weitere mögliche Gründe dafür anführt, warum die epigraphische Evidenz vor Ort in keinster Weise dem Pausaniastext entspricht. Daß die besagte Episode in Pausanias, Graeciae descriptio 5.20.8 dennoch überliefert ist, liegt m.E. nur darin begründet, daß der Schriftsteller die Beschreibung der Oinomaossäule, die das eigentliche Thema des Kapitels ist, durch eine interessante Begebenheit auskleiden wollte.

${ }^{20}$ Damit ist natürlich die Variante mit zwei verschiedenen Monumenten nicht ganz ausgeschlossen. Wenn jedoch die vorgestellte Version zuträfe, so wäre das Siegermonument von Olympia keineswegs das einzige Monument, das für Minicius Natalis nach seinem Prokonsulat errichtet wurde, oder das er selbst in Auftrag gab; so wurden ihm etwa CIL II $4510=\operatorname{IRB} 31=\mathbb{I R C} 32$ und CIL II $4511=\operatorname{IRB} 32$ $=$ IRC IV 33 frühestens 154/155 dediziert.

${ }^{21}$ Pausanias, Graeciae descriptio 5.20.8. 
befreundeten Senator Ti. Claudius Atticus Herodes und seine Frau Annia Regilla vollzogen worden war. ${ }^{22}$

Die ,Olympia-Episode' ist nicht zuletzt deshalb von Interesse, weil sie die einzige nachgewiesene Verbindung beider Minicii Natales zum Osten des römischen Reiches aufzeigt. Niemals vor oder nach dem Sieg haben Vater und Sohn Ämter in den östlichen Provinzen bekleidet, niemals - nach allem, was wir wissen - Kontakte dorthin gepflegt. ${ }^{23}$ Man könnte spekulieren und fragen, ob es dann als ein Zufall zu werten ist, daß der jüngere Minicius Natalis in dem Jahr mit seiner Quadriga in Olympia vertreten war, in dem Kaiser Hadrian von den Athenern den Titel 'O $\lambda$ ú $\mu \pi$ เos erhielt. ${ }^{24}$

Der Philhellene Hadrian, beseelt von dem Gedanken an ein großes panhellenisches Programm, hatte sich bereits um 111 und 124/125 in Griechenland aufgehalten; bei seinem ersten Besuch hatte er das Bürgerrecht und das Archontat der Stadt Athen erhalten, beim zweiten Besuch, in dessen Verlauf er wohl auch Olympia streifte und die dortige Statue des Zeus Olympios restaurieren ließ, war er u.a. in die eleusinischen Mysterien eingeführt worden. ${ }^{25}$ Kontakte zu ,griechischen' Senatoren wie dem jungen Ti. Claudius Atticus Herodes wurden intensiviert, nicht zuletzt durch eine

\footnotetext{
${ }^{22}$ Dokumentiert durch IOlymp. 610-626. Regilla erscheint als offizielle Stifterin der Exedra (IOlympia 610). Dazu W. Ameling, Herodes Atticus (Hildesheim 1983) I, 91ff. und II, 127f. Vgl. ferner P. Graindor, Un milliardaire antique. Hérode Atticus et sa famille (Kairo 1930), 85ff. und $191 \mathrm{ff}$. (mit Abbildungen) sowie die Abbildungen bei Herrmann 1972, a.a.O. (Anm. 5), 191 ff. Es wird vielfach in der Forschung angemerkt, daß Pausanias dieses Bauwerk in der ,Beschreibung Griechenlands' verschweigt. Die Gründe hierfür dürften sowohl in der oben angesprochenen Prioritätensetzung des Autors (vgl. Anm. 19) als auch darin liegen, daß er das imposante Bauwerk offenbar als für Olympia unpassende ,Luxusarchitektur' verschmähte. Vgl. die Forschungsdiskussion bei Habicht 1985, 137f., Anm. 74. Quellenbefund und Literatur zu Herodes Atticus sind sehr umfangreich. Es sollte daher genügen, hier auf $\mathrm{H}$. Halfmann, Die Senatoren aus dem östlichen Teil des Imperium Romanum bis zum Ende des 2. Jh. (Göttingen 1979), 155ff., Nr. 68, zu verweisen, der alle bis dahin bekannten Quellenbelege und wissenschaftlichen Beiträge bequem auflistet. Ergänzend seien Ameling 1983, a.a.O., I und für einige neuere Erkenntnisse (etwa bezüglich der Laufbahn) A.R. Birley, 'Hadrian and Greek Senators', Zeitschrift für Papyrologie und Epigraphik 116 (1997), 209ff., bes. 229ff., genannt.

${ }^{23}$ Mit einer kleinen Ausnahme: Moesia inferior, wo sich der jüngere Minicius Natalis um 115 als Militärtribun und ca. 142-144 als Statthalter aufhielt, war teilweise griechisch. Vgl. A.R. Birley, 'Some Governors of Moesia inferior', in: G. Fülöp (Hg.), Festschrift für Jenö Fitz. Jenö Fitz septuagenario 1991 (Skékesfehérvar 1996), 49f.

${ }^{24}$ Vgl. Birley 1997, 219f. und W. Weber, Untersuchungen zur Geschichte des Kaisers Hadrianus (Leipzig 1907), 209ff. zur Verleihung des Titels und den Umständen. Seit 129 firmierte er im Osten unter diesem Titel.

${ }^{25}$ Vgl. Birley 1997, 58ff. und 175ff. Die genaue Chronologie der Reisen Hadrians ist weiterhin ein Gegenstand intensiver wissenschaftlicher Diskussion. Dazu auch H. Halfmann, Itinera principum. Geschichte und Typologie der Kaiserrreisen im Römischen Reich (Stuttgart 1986), 188ff. sowie A.R. Birley, 'Hadrian's Travels' in der vorliegenden Publikation. Daß Hadrian Olympia besuchte, macht Birley 1997, 181 sehr wahrscheinlich.
} 
Förderung der Karrieren. ${ }^{26}$ Nun kehrte er 128/129 nach Griechenland zurück, nicht nur, um wiederum nach Eleusis zu reisen, sondern sicherlich auch, um die von ihm initiierte Vollendung des Zeus Olympios-Tempels in Athen zu sehen und den oben erwähnten Titel anzunehmen, der de facto im Osten des Reiches zu einer Gleichsetzung mit Zeus führte. ${ }^{27}$ In Hadrians großem Gefolge dürfte sich bei dieser Griechenlandreise auch L. Minicius Natalis Quadronius Verus, sein quaestor Augusti aus dem Jahr 121, befunden haben, der dann wenig später Richtung Olympia weiterzog, während Hadrian nach Kleinasien reiste. ${ }^{28}$

Die Besucher der 227. Olympiade des Jahres 129 sahen in dem Senator Minicius Natalis in jedem Fall einen Repräsentanten der römischen Macht. Er konnte zwar seinen Sieg, der im übrigen einzigartig ist, ${ }^{29}$ nicht einplanen, aber dennoch erfüllte es ihn sicherlich mit einem gewissen Stolz, diesen Sieg als Vertreter Roms davongetragen zu haben und im Anschluß im Tempel des Gottes geehrt worden zu sein, mit dem der Kaiser sich so eng verbunden fühlte. Interessant ist, daß er dieses besondere Ereignis in seinem Leben nach dem heutigen Erkenntnisstand fast eine Generation später - lange nach Hadrians Tod - lediglich an dem Ort des Geschehens herausstellte. Adressaten des Monuments konnten somit nur die Menschen sein, die die Altis von Olympia besuchten, also etwa Athleten, Zuschauer oder Pilger ${ }^{30}$ Alles in allem bleibt zunächst unklar, ob der jüngere Minicius Natalis durch die statuarische Nähe zu Zeus Olympios persönliche Nähe zu Hadrian demonstrieren wollte. ${ }^{31}$ Einige Bemerkungen zu weiteren Verbindungen zwischen dem Senator und dem Kaiser könnten ein wenig Licht in das Dunkel bringen.

\footnotetext{
${ }^{26}$ Vgl. Birley 1997, a.a.O. (Anm. 22), 229ff. sowie Birley 1997, 63, 89, 177 und 217. Zu Herodes Atticus' Vater vgl. auch Halfmann 1979, a.a.O. (Anm. 22), 120ff., Nr. 27.

${ }^{27}$ Vgl. Birley 1997, 215ff. und Weber 1907, a.a.O. (Anm. 24), 208ff. mit Anm. 739. Jüngst hat W. Eck (W. Eck, D. MacDonald \& A. Pangerl, 'Neue Diplome für das Heer der Provinz Syrien', Chiron 32 [2002], 434ff.) versucht zu zeigen, daß Hadrian erst 129 nach Griechenland abgereist ist, da er 128 in den Diplomen nicht als Prokonsul bezeichnet wird. Anders A.R. Birley in diesem Band.

${ }^{28}$ So auch Birley 1997, 216.

${ }^{29}$ Eine rasche Durchsicht der einschlägigen Inschriftenpublikationen und oben zitierten Listen von Herrmann und Moretti (vgl. Anm. 8) ergab, daß L. Minicius Natalis Quadronius Verus bis jetzt der einzige uns bekannte Senator der Kaiserzeit ist (außerhalb der Kaiserfamilien), der in einem olympischen Wagenrennen den Sieg errang.

${ }^{30}$ Man hätte vielleicht ein weiteres Ehrenmonument in Barcino, der patria der Minicii Natales, erwarten können.

${ }^{31}$ Die Oinomaossäule, in deren Nähe sich das minicische Monument offenbar befand (nach Pausanias, Graeciae descriptio 5.20.8), stand nur wenige Meter nordöstlich des Zeus Olympios-Tempels.
} 
Zwei Familien dominierten in der Hohen Kaiserzeit die in der Tarraconensis gelegene Stadt Barcino, die Pedanii und die Minicii Natales, wobei keineswegs völlig sicher ist, daß die senatorischen Pedanii von dort stammten, da wir lediglich indirekte Zeugnisse - wie etwa eine allgemein starke Abundanz von Pedanii vor Ort - besitzen. Da aber vice versa bis jetzt Gegenbeweise fehlen, hat sich die Forschung mehr oder weniger darauf geeinigt, Barcino als patria zu favorisieren. ${ }^{32}$ Ronald Syme, der ebenfalls für diese Lösung plädierte, ${ }^{33}$ bemerkte jedoch: „Between Minicii and Pedanii no links of blood or propinquity can be discovered.“34 Seine auf den vorliegenden Fakten beruhende Feststellung läßt Spielraum für prosopographische Spekulationen, vorausgesetzt, man akzeptiert die These zweier bedeutender senatorischer Familien in Barcino, was in der Folge geschehen soll. Unter dieser Prämisse kann man weiter fragen, ob möglicherweise doch verwandtschaftliche Beziehungen zwischen den Pedanii und den Minicii Natales existierten: Dies wäre keineswegs unmöglich, zumal man bis heute weder den Namen der Mutter des älteren Minicius Natalis noch den der Frau seines Sohnes kennt.

L. Minicius Natalis Quadronius Verus wurde um 96 in Barcino, der patria seiner Familie, geboren. ${ }^{35}$ Der Name seiner Mutter ist nicht

\footnotetext{
${ }^{32}$ Die Diskussion über die Herkunft der senatorischen Pedanii führt in extenso Caballos Rufino 1990 I,2, 416f. (mit weiterer Literatur). Er sortiert die Familie unter Senatores incerti et incerta origine ein. In der Tat sind die Indizien, die für Barcino sprechen könnten, nicht sehr gewichtig, da ausnahmslos indirekter Art; es fehlen direkte Quellenbelege. Neben dem m.E. einzigen überzeugenden Argument, nämlich den zahlreichen inschriftlichen Belegen für Personen mit dem nomen gentile Pedanius in Barcino, nennt Caballos Rufino äußerst unsichere Punkte, wie etwa die familiären Kontakte der Pedanii zu dem vielleicht aus Spanien stammenden L. Iulius Ursus Servianus oder den gemeinsamen ordentlichen Konsulat des Cn. Pedanius Fuscus Salinator mit Kaiser Hadrian im Jahre 118. Insofern besitzen die Bedenken, die insbesondere P. Le Roux ('Les sénateurs originaires de la province d'Hispania citerior au Haut-Empire romain', in: Epigrafia e ordine senatorio II [Rom 1982], 448f.) vorbringt, eine gewisse Relevanz. Auch K. Wachtel (PIR ${ }^{2}$ P 202) möchte Le Roux' These nicht gänzlich ablehnen, scheint aber das Votum der älteren Forschung für Barcino zu bevorzugen.

${ }_{33}$ Vgl. etwa R. Syme, Tacitus II (Oxford 1958), 785; ders., 'Rival Cities, notably Tarraco and Barcino', Ktèma 6 (1981), 280 = Roman Papers IV, 87 und 282 = 89f.; ders., 'More Narbonensian Senators', Zeitschrift für Papyrologie und Epigraphik 65 (1986), 14 = Roman Papers VI, 222.

${ }^{34}$ Syme 1981/1988, a.a.O. (Anm. 33), $284=91$.

${ }^{35} \mathrm{CIL}$ II $4511=$ IRB $32=$ IRC IV 33. Dort wird nicht nur deutlich, daß Minicius Natalis in Barcino geboren wurde, sondern auch, daß dies im Januar oder Februar geschah. Das genaue Geburtsjahr ist unsicher; es hängt von der Datierung der später bekleideten Ämter ab. In der Forschung herrscht diesbezüglich Uneinigkeit: E. Groag (Groag 1932, 1837) denkt an 97, weil er die Quästur sehr spät datiert (123), ebenso R. Etienne (Etienne 1965, a.a.O. [Anm. 3], 72). Die PIR ( $\mathrm{PIR}^{2} \mathrm{M}$ 620) nimmt das Jahr 96 an, ausgehend von einer Bekleidung der Quästur um das Jahr 121. So auch J. Fitz, Die Verwaltung Pannoniens in der Hohen Kaiserzeit. Bd. II (Budapest 1993), 666. Caballos Rufino 1990 I,1, 228, spricht von ,probablemente del año 96 o 97“, kann sich also nicht definitiv entscheiden. M.E. ist die frühere Datierung zu präferieren; Minicius Natalis hätte dann mit 18 sein erstes Amt bekleidet
} 
überliefert, doch ist es nicht unwahrscheinlich, daß sie zu den Quadronii gehörte: Sein Vater führte 106 gemeinsam einem Landsmann namens Q. Licinius Silvanus Granianus, ${ }^{36}$ einem Tarraconenser, die fasces als Suffektkonsul. Es ist auffallend, daß ihre Söhne, L. Minicius Natalis Quadronius Verus und Q. Licinius Silvanus Granianus Quadronius Proculus, ${ }^{37}$ beide das äußerst seltene nomen gentile Quadronius trugen. ${ }^{38}$ Somit liegt die Vermutung nahe, daß sie miteinander verwandt waren, wahrscheinlich über die mütterliche Seite: Ronald Syme konnte überzeugend darlegen, daß jeder von beiden Senatoren wohl eine Mutter namens Quadronia hatte und diese Schwestern waren. ${ }^{39}$ Zum gemeinsamen Konsulat der Väter schreibt er: „The consulships of Natalis and Silvanus reflect (it should seem) the influence of Licinius Sura." ${ }^{40}$ L. Licinius Sura (cos. suff. 97 (?); cos. II ord. 102; cos. III ord. 107) aus der Tarraconensis, engster Vertrauter Trajans (dessen Wurzeln, wie die Hadrians, ebenfalls in Italica lagen), fungierte möglicherweise als Patron einiger Senatoren spanischer Herkunft, u.a. des älteren Minicius Natalis. ${ }^{41}$ Über diese Verbindung dürfte in der Folge ein sehr enges

(tresvir monetalis im Jahre 114) und wäre später, im Alter von 24 Jahren, als candidatus principis für die Quästur designiert sowie kurz vor seinem 25. Geburtstag in dieses Amt eingesetzt worden.

${ }^{36} \mathrm{Zu}$ Q. Licinius Silvanus Granianus vgl. PIR ${ }^{2}$ L 247; A. Stein, 'Licinius', RE XIII (Stuttgart 1926), 459; Caballos Rufino 1990 I,1, 180ff., Nr. 101.

${ }^{37} \mathrm{Zu}$ Q. Licinius Silvanus Granianus Quadronius Proculus vgl. PIR ${ }^{2}$ L 249; E. Groag, 'Licinius', RE XIII (Stuttgart 1926), 459ff;; A. Stein, 'Licinius', RE Suppl. VII (Stuttgart 1940), 418; Caballos Rufino 1990 I, 1, 182f., Nr. 102.

${ }^{38}$ Die PIR kennt neben diesen beiden nur noch einen Senator mit dem Namensbestandteil Quadronius: T. Iulius Maximus Manlianus Brocchus Servilianus A. Quadronius [Verus?] L. Servilius Vatia Cassius Cam[ars?] aus Nemausus (PIR ${ }^{2}$ I 426; E. Groag, 'Iulius', RE X [Stuttgart 1918], 678f.). Aufgrund der Seltenheit des nomen gentile ist m.E. davon auszugehen, daß dieser Suffektkonsul von 112, der vermutlich aus Nemausus stammte und Patron von Calagurris in der Tarraconensis war (vgl. etwa $\mathrm{CL}$ XII 3167 = ILS 1016), mit den anderen Quadronii verwandt war, sehr wahrscheinlich durch Adoption. Ähnlich Le Roux 1982, a.a.O. (Anm. 32), 448. Drei Quadronii aus Narbo (CIL XII 4414. 4415. 5081) waren offenbar Freigelassene dieses Mannes.

${ }^{39}$ R. Syme, 'Pliny the Procurator', Harvard Studies in Classical Philology 73 (1969), $233=$ Roman Papers II, 770, nahm zunächst nur eine verwandtschaftliche Beziehung an; später präzisierte er diese These, indem er die Vermutung äußerte, die beiden Konsuln von 106 hätten jeder eine Quadronia geheiratet: Syme 1981/1988, a.a.O. (Anm. 33), 282 = 89; 'Spaniards at Tivoli', Ancient Society 13/14 (1982/83), 247 = Roman Papers IV, 99f:; 'Clues to testamentary adoption', Epigrafia e Ordine Senatorio I (Rom 1982), 403 = Roman Papers IV, 165. Zustimmend O. Salomies, Adoptive and Polyonymous Nomenclature in the Roman Empire (Helsinki 1992), 141f. und Caballos Rufino 1990 $\mathrm{I}, 1,183$ und 228 .

${ }^{40}$ Syme 1969/1979, a.a.O. (Anm. 39), $233=770$.

${ }^{41}$ So die Vermutung von R. Syme (Syme 1981/1988, a.a.O. [Anm. 33], $283=91$ ): „Minicius Natalis belonged to the faction of Sura, such is the assumption." Vgl. ders., 'Hadrian and the Senate', Athenaeum 62 (1984), 37f. = Roman Papers IV, 302. Zu Licinius Sura vgl. PIR ${ }^{2}$ L 253; E. Groag, 'Licinius', RE XIII (Stuttgart 1926), 471ff.; Caballos Rufino 1990 I,1, 183ff. Eine Verbindung dieses Senators zu den senatorischen Pedanii, deren Herkunft aus Barcino, wie bereits erwähnt wurde, 
Verhältnis zum Kaiserhaus gewachsen sein, welches sich nicht zuletzt evident durch das Engagement des L. Minicius Natalis im ersten Dakerkrieg $(101 / 102)$ und seine spätere langjährige Statthalterschaft in der für Rom militärisch wichtigen Grenzprovinz Pannonia superior manifestierte. ${ }^{42}$

Eine andere „spanische" Senatorenclique in Rom führte der ebenfalls sehr einflußreiche L. Iulius Ursus Servianus (cos. suff. 90; cos. II ord. 102; cos. III ord. 134), ${ }^{43}$ der über seine Tochter, die Cn. Pedanius Fuscus Salinator (cos. ord. 118) geheiratet hatte, ${ }^{44}$ nicht nur mit den Pedanii, sondern als Hadrians Schwager auch mit den Aelii aus Italica verwandtschaftlich verbunden war. ${ }^{45}$ Beide Rivalen, Servianus und Sura, bekleideten 102 gemeinsam den ordentlichen Konsulat. Als letzterer im Jahre 108 starb, dürften dessen Schützlinge in Hadrian einen neuen Patron gefunden haben. ${ }^{46}$ Die Beziehungen der Minicii Natales zum Kaiser waren in der Folgezeit offenbar gut; zumindest gibt es keine Hinweise für das Gegenteil. Sie konnten nicht nur bei der Durchführung der Amtsgeschäfte in Rom intensiviert werden, sondern auch in Phasen der Erholung im nahen Tibur, wo neben anderen senatorischen Familien aus Spanien auch die Aelii und die Minicii Natales begütert waren und der Olympiasieger nach 139 als Patron fungierte. ${ }^{47}$ In diesem Zusammenhang sei erwähnt, daß einige Votiv-

unsicher ist (vgl. dazu Anm. 32), ist nicht nachweisbar.

${ }^{42} \mathrm{~L}$. Minicius Natalis ging hochdekoriert aus dem Krieg hervor; vgl. dazu nur die Inschriften CIL II 4509

$=6145=\operatorname{ILS} 1029=\operatorname{IRB} 30=\operatorname{IRC}$ IV 30 (Barcino) und CIL VI $31739=41109$ (Rom) mit den Hinweisen auf diverse dona militaria. Zum Statthalterposten in Pannonia superior vgl. zuletzt Fitz 1993, a.a.O. (Anm. 35), 474 und Birley 1997, 89. Die epigraphische Evidenz zeigt jetzt, daß der ältere Minicius Natalis mindestens von 112 bis in die hadrianische Zeit hinein Statthalter der Provinz war. Prof. Dr. W. Eck (Köln) danke ich in diesem Zusammenhang für den Hinweis auf neue Militärdiplome.

${ }^{43}$ Zu Iulius Ursus Servianus vgl. PIR ${ }^{2}$ I 631 und 569; E. Groag, 'Iulius', RE X (Stuttgart 1918), 882ff.; Caballos Rufino 1990 I,2, 386ff. Seine Herkunft ist sehr unsicher; sowohl eine spanische als auch eine narbonensische patria werden diskutiert.

${ }^{44} \mathrm{Zu}$ Pedanius Fuscus Salinator vgl. PIR ${ }^{2}$ P 200; E. Groag, 'Pedanius', RE XIX (Stuttgart 1937), $21 \mathrm{ff}$; Caballos Rufino 1990 I 2, $418 f$., Nr. I 46.

${ }^{45}$ Das komplexe Beziehungsgeflecht zwischen Sura, Servianus, Nerva, Trajan und Hadrian beschreibt R. Syme, 'Hadrian's Autobiography: Servianus and Sura', Bonner Historia-Augusta-Colloquium 1986 (Bonn 1991), 189ff. = Roman Papers VI, 398ff.

${ }^{46}$ So Syme 1984/1988, a.a.O. (Anm. 41), 37f. = 302 und Birley 1997, 54 .

${ }^{47}$ Wenn Rom für die Senatoren das negotium symbolisierte, so stand eine Stadt wie Tibur für das otium. Ronald Syme (Syme 1983/1988, a.a.O. [Anm. 39], 241 = 94) beschreibt sie folgendermaßen: „The charm of Tibur is on abundant testimony: the fresh air, the cascades of the Anio, the groves and the orchards." Die Erkenntnis, daß dort vor allem Senatoren aus dem spanischen Raum beguttert waren, verdanken wir ebenfalls Syme 1983/1988, a.a.O. (Anm. 39), 241ff. $=94 \mathrm{ff}$.). Vgl. dazu auch Birley 1997, 192f. Zum Grundbesitz der Minicii Natales vgl. ausführlich A.M. Andermahr, Totus in praediis. Senatorischer Grundbesitz in Italien in der Frühen und Hohen Kaiserzeit (Bonn 1998), 342f. Den Patronat notieren L. Harmand, Le patronat sur les collectivités publiques des origines au basempire (Paris 1957), 225 und R. Duthoy, 'Le profil social des patrons municipaux en Italie sous le 
inschriften auf Marmorbasen aus Tibur und aus anderen Städten Italiens erhalten sind, die eine besondere Vorliebe des jüngeren Minicius Natalis für mehrere, vornehmlich östliche Gottheiten, nämlich Hercules Victor (Schutzgott von Tibur), Asklepios, Helios, Sarapis und Isis, aufzeigen. ${ }^{48}$ Das muß umso mehr erstaunen, als der „westliche“ Senator, der außer bei seinem Olympiasieg nie im Osten weilte, solch ein lebhaftes Interesse für Götter aus diesem Raum entwickelte.

Bemerkenswert ist gleichfalls, daß - nach allem, was wir bisher wissen - L. Minicius Natalis Quadronius Verus und Hadrian die einzigen beiden Senatoren der römischen Kaiserzeit waren, die drei Militärtribunate in Folge innehatten, Hadrian zwischen 95 und 98, Minicius Natalis zwischen ca. 115 und $117 .^{49}$ Man kann diese außergewöhnliche Ämterkonstellation wohl als einen puren Zufall werten, doch scheint es, daß der Senator aus Barcino alle drei Tribunate bei Legionen im Donauraum innehatte, den letzten um 117 bei seinem Vater in Pannonia superior, der dort damals, wie bereits angedeutet wurde, Statthalter war. ${ }^{50}$ Letztendlich wird der Kaiser

Haut-Empire', Ancient Society 15-17 (1984-1986), 145, Nr. 223, auf der Grundlage von CIL XIV $3599=\Pi$ LS $1061=$ Inscr. Ital. 4,1,113. Zu Hadrians Villa in Tibur vgl. etwa H. Kähler, Hadrian und seine Villa bei Tivoli (Berlin 1950) und M.T. Boatwright, Hadrian and the City of Rome (Princeton 1987), $138 \mathrm{ff}$.

${ }^{48}$ CIL XI 2925 = ILS 3221 (Volci); IGR I 1391 (Minturnae); CIL XIV $3554=\mathbb{L S} 3415=$ Inscr. Ital. 4,1,56. $3599=$ ILS $1061=$ Inscr. Ital. 4,1,113. $3600=$ Inscr. Ital. 4,1,1 14; IG XIV $1125=$ IGR 1,376= Inscr. Ital. 4,1,33 (alle Tibur). Minicius Natalis' besondere Beziehung zu Tibur wird auch durch das Amt des curator fani Herculis Victoris dokumentiert, das er nachweislich innehatte (CIL XIV 3599= ILS 1061 = Inscr. Ital. 4,1,113). Vergleichbar ist der fast gleichaltrige P. Mummius Sisenna Rutilianus (consul suffectus 146), der wohl ebenfalls aus Spanien stammte, auch patronus von Tibur und curator fani Herculis Victoris ebendort war (CIL XIV $3601=$ LS $1101=$ Inscr. Ital. 4,1,115). Vgl. Andermahr 1998, a.a.O. (Anm. 47), 346 mit weiterer Literatur.

${ }^{49}$ Dazu zuletzt Birley 1997, 37: „Hadrian's third military tribunate is unparalleled [...]. But the circumstances in 97 were very special: Hadrian was now, after all, the nearest male kinsman of the heir to the throne [sc. Trajan]." Vgl. auch R. Syme, 'Domitian: The last Years', Chiron 13 (1983), 141 = Roman Papers IV, 272.

${ }^{50}$ Beobachtet von R. Syme, 'A Dozen early Priesthoods', Zeitschrift für Papyrologie und Epigraphik 77 (1989), 247f. = Roman Papers VI, 425. Vgl. zu dem Sachverhalt auch Fitz 1993, a.a.O. (Anm. 35), 663ff. und Anm. 42. Es kann hier nicht der Ort sein, die gesamte Laufbahn des L. Minicius Natalis Quadronius Verus neu zu diskutieren; dies wurde an anderer Stelle geleistet, z.B. von Caballos Rufino 1990 I,1, 228, der zahlreiche Untersuchungen bequem zusammenfaßt. Dennoch seien ein paar kurze Bemerkungen zu den Tribunaten erlaubt: Der jüngere Minicius Natalis fungierte um 115 als Militärtribun der legio I Adiutrix, die unter dem Kommando des baetischen Legionslegaten A. Platorius Nepos, eines langjährigen Freundes und Landsmannes Hadrians (vgl. Historia Augusta, Vita Hadriani 4.1f.), stand. Der exakte Aufenthaltsort der Legion (und damit auch des Minicius Natalis) um 115 ist unsicher, einige plädieren für Dacia, andere meinen, daß sie in den Partherkrieg involviert war (vgl. Birley 1981, a.a.O. (Anm. 4), 103). Bei den in Dacia nachgewiesenen Soldaten der legio I Adiutrix könnte es sich aber auch lediglich um eine Vexillation gehandelt haben. So die plausible Erklärung von I. Piso, Fasti provinciae Daciae, Band I: Die senatorischen Amtsträger (Bonn 1993), 
spätestens jetzt auf seinen jungen „spanischen“ Landsmann aufmerksam geworden sein. Ein Zusammentreffen in Barcino, das Hadrian auf seiner Reise nach Tarraco zu Beginn des Jahres 123 sicherlich besuchte, ist eher unwahrscheinlich, da der jüngere Minicius Natalis zu dieser Zeit wohl den Volkstribunat in Rom innehatte. ${ }^{51}$ Dessen ungeachtet könnte aber sein Vater, der seine Ämterlaufbahn 122 mit dem Prokonsulat in Africa proconsularis beendet hatte, den Kaiser empfangen und ihm die imposante minicische Thermenanlage vorgeführt haben. ${ }^{52} \mathrm{Cn}$. Pedanius Fuscus Salinator und seine Frau lebten damals wohl noch und könnten ebenfalls vor Ort gewesen sein, wahrscheinlich als Hadrians Reisebegleitung. Der Senator, der fünf Jahre zuvor den ordentlichen Konsulat mit dem Onkel seiner Frau bekleidet hatte, wäre zu diesem Zeitpunkt ohnehin der perfekte Erbe für Hadrian gewesen. ${ }^{53}$

Es wurde lange Zeit gerätselt, warum der Kaiser L. Minicius Natalis Quadronius Verus vor seinem Tod im Jahre 138 nicht mehr zum Konsulat verholfen hat, zumal dieser candidatus principis für Quästur (120/121) und Volkstribunat (122/123) gewesen war und als quaestor Augusti engsten Kontakt zum Kaiser gehabt hatte, nicht nur in Rom, sondern sicherlich auch auf einigen der Reisen, die der Kaiser fortan unternahm. ${ }^{54}$ Eine plausible

7f., der die unterschiedlichen Meinungen gegenüberstellt. Um 116 hatte Minicius Natalis dasselbe Amt bei der legio XI Claudia in Moesia inferior (unter dem Statthalter Q. Pompeius Falco) inne und um 117 bei der legio XIV Gemina in Pannonia superior (wohl unter seinem Vater als Statthalter und dem Legionslegaten Sex. Iulius Severus). Wenn sich der junge Minicius Natalis um 115 wirklich im Osten aufhielt, so wäre natürlich Symes oben zitierte Beobachtung nicht haltbar. Die Chronologie der Militärtribunate ist insgesamt sehr spekulativ und hypothetisch. Es wäre z.B. zu fragen, ob jeder der drei Tribunate wirklich nur ein Jahr dauerte.

${ }^{51}$ Ein Besuch Hadrians in Barcino ist schon wegen seiner verwandtschaftlichen Beziehungen zu den Pedanii mehr als wahrscheinlich. Vgl. Birley 1997, 146f. zur Reise nach Spanien.

${ }^{52}$ Mit CIL II $4509=6145=$ ILS $1029=$ IRB $30=$ IRC IV 30 ist die Bauinschrift des balineum der Minicii Natales in Barcino auf uns gekommen. Der jüngere Minicius Natalis erscheint dort als tribunus plebis designatus, was bedeutet, daß die Anlage kurz vor Hadrians Besuch oder sogar aus Anlaß seiner Ankunft in Barcino eingeweiht wurde. Vgl. dazu W. Eck und F.J. Navarro, 'Das Ehrenmonument der Colonia Carthago für L. Minicius Natalis Quadronius Verus in seiner Heimatstadt Barcino', Zeitschrift für Papyrologie und Epigraphik 123 (1998), 237ff. und A. Krieckhaus, Fallstudien zu senatorischen Familien und ihren Heimatstädten im 1. und 2. Jahrhundert n.Chr. (in Vorbereitung).

${ }^{53}$ So Birley 1997, 146. Pedanius Fuscus Salinator und seine Frau sind ab 118 nicht mehr belegt, wie schon Ronald Syme feststellen mußte (R. Syme, 'The Ummidii', Historia 17 (1968), 87 = Roman Papers II, 674 und ders., 'Ummidius Quadratus, capax imperii', Harvard Studies in Classical Philology 82 (1979), 290 = Roman Papers III, 1160). Vgl. PIR P 200; Birley 1997, 201f. und 215; Caballos Rufino 1990 I,2, 418. Dennoch ist es m.E. mehr als wahrscheinlich, daß sie 123 noch lebten und erst in den späteren zwanziger Jahren starben.

${ }^{54}$ Eine Ausnahme dürfte Hadrians Reise in den Nordwesten darstellen, die im Frühjahr 121 begann: Offenbar verzichtete der Kaiser (zunächst) auf seine beiden quaestores Augusti: Es ist nämlich epigraphisch bezeugt, daß der jüngere Minicius Natalis, während er noch die Quästur bekleidete, in 
Erklärung hat Werner Eck geliefert: „Ganz offensichtlich trat nach einer anfänglichen Förderung des jungen Senators durch Hadrian in den späten Regierungsjahren dieses Kaisers eine Abkühlung in den guten Beziehungen ein. Denn ansonsten hätte L. Minicius Natalis Quadronius Verus früher zum Konsulat kommen müssen.“55 Diese „Abkühlung“ ist zeitlich nur schwer greifbar; bis zur Prätur (127) ist keine Verzögerung in der Laufbahn festzustellen. Danach vergingen jedoch deutlich mehr als 10 Jahre bis zum Konsulat im Jahre 139 - eine sehr lange Zeit für einen ehemaligen candidatus principis und Sohn eines sehr einflußreichen Senators, der es bis zur Statthalterschaft von Africa proconsularis gebracht hatte. ${ }^{56}$

Man könnte also geneigt sein, irgendwann in der Zeit zwischen 127 und 138 einen Bruch in der schon fast traditionell engen Beziehung der Minicii Natales zum Kaiserhaus zu vermuten. Dies führt uns zu den Pedanii zurück: Wenn der jüngere Minicius Natalis eine Pedania geheiratet hätte, vielleicht eine Tochter oder Schwester des Cn. Pedanius Fuscus Salinator, dann bestünde die Möglichkeit, daß die Verweigerung des Konsulats suo anno mit den Ereignissen, die sich im Zuge der Adoption des L. Ceionius Commodus durch den mittlerweile erkrankten Hadrian 136/137 zutrugen, zusammenhängt: L. Iulius Ursus Servianus und sein Enkel Fuscus, ganz offensichtlich der Sohn des ordentlichen Konsuls von 118 und der Nichte Hadrians, waren mit der Entscheidung des Kaisers nicht einverstanden und planten wohl einen Umsturz, der jedoch scheiterte und beider Leben

Africa proconsularis als Legat an der Seite seines Vaters, der um 121/122 den Prokonsulat der Provinz innehatte, tätig wurde (vgl. Thomasson 1996, a.a.O. (Anm. 11), 62 und 107, Nr. 26 mit Quellenhinweisen). Der uns namentlich nicht bekannte Amtskollege des Minicius Natalis verweilte vermutlich in Rom beim Senat. Der Grund dafür könnte darin liegen, daß der ab epistulis $\mathrm{C}$. Suetonius Tranquillus - neben dem praefectus praetorio C. Septicius Clarus - mit Hadrian unterwegs war. So die These von Syme 1989/1991, a.a.O. (Anm. 50), 247f. = 425; ders., 'The Travels of Suetonius Tranquillus', Hermes 109 (1981), 110 = Roman Papers III, 1342; ders., Hadrianic Proconsuls of Africa, Zeitschrift für Papyrologie und Epigraphik 37 (1980), 6 = Roman Papers III, 1307; ders. 1958, a.a.O. (Anm. 33), 779f. So auch Birley 1997, 115 und ferner ders. 1981, a.a.O. (Anm. 4), 365ff. (zu Septicius Clarus und Sueton).

${ }_{55}^{5}$ W. Eck 1974, a.a.O. (Anm. 3), 283.

${ }^{56}$ Zum Konsulat: AE 1955,17 = CL XVI 175. Minicius Natalis' Amtskollege, dessen Name im genannten Militärdiplom nur zum Teii erhalten ist, war mit großer Wahrscheinlichkeit L. Claudius Proculus Cornelianus, der möglicherweise ebenfalls aus dem spanischen Raum stammte ( $\mathrm{PIR}^{2} \mathrm{C}$ 979/980). So bereits G. Alföldy, Konsulat und Senatorenstand unter den Antoninen. Prosopographische Untersuchungen zur senatorischen Führungsschicht (Bonn 1977), 138f. und 322 sowie Caballos Rufino 1990 I 2, 344f., Nr. I 9. Zur Dauer einer prätorischen Laufbahn vgl. grundlegend W. Eck, 'Beförderungskriterien innerhalb der senatorischen Laufbahn, dargestellt an der Zeit von 69 bis 138 n.Chr.', in: ANRW II 1, $181 \mathrm{ff}$. = 'Criteri di avanzamento nella carriera senatoria (69-138 d.C.)', in: W. Eck, Tra epigrafia prosopografia e archeologia, Scritti scelti, rielaborati ed aggiornati (Rom 1996), 36ff. (mit Ergänzungen). 
kostete.$^{57}$ Dieser Coup d'État muß negative Auswirkungen auf die Beziehung Hadrians zu den nächsten Verwandten der Verschwörer gehabt haben, die natürlich auch seine (angeheirateten) Verwandten waren. Unter den oben geschilderten Voraussetzungen könnten L. Minicius Natalis Quadronius Verus und seine Familie sehr wohl davon betroffen gewesen sein. ${ }^{58}$ Wie auch immer - nachdem der neue Kaiser Antoninus Pius den Thron bestiegen hatte, gehörte der Senator aus Barcino zu den ersten, denen die Konsulwürde zuteil wurde, was $m$.E. ein untrügliches Zeichen dafür ist, daß seine Familie damals - neben den mit Hadrian eng verbundenen baetischen Annii Veri - zu den wichtigsten senatorischen Familien aus dem spanischen Raum zählte.

\section{III}

„He paid ambitious honour to old Hellas by winning a chariot race at Olympia. In Italy he worshipped Hercules at Tibur - but also Aesculapius. At another town, however, it was Serapis and Isis ,of the myriad names"." 59 So faßt Ronald Syme die „östlichen“ Aktivitäten des jüngeren Minicius Natalis knapp zusammen. Auch Hadrian kam auf seinen Reisen durch den Osten des römischen Reiches mit den erwähnten Gottheiten in Kontakt, auch Hadrian hatte eine besondere Beziehung zu Zeus Olympios, auch Hadrian liebte alles Griechische. Man kann festhalten, daß L. Minicius Natalis Quadronius Verus bei seinem Auftritt in Olympia, das im übrigen durch seine jahrhundertealte Tradition in gewisser Weise eine Art Symbol für das alte Griechenland darstellte, nicht nur einen Sieg für sich selbst, sondern auch für das Imperium Romanum davontrug und eben diesen Staat und seinen eigenen Stand, den ordo senatorius, dort repräsentierte. Hier nun aber von einer Art ,Nacheiferung' des Kaisers zu sprechen, würde zu weit gehen, zumal ein starkes Interesse für griechische Sprache, Philosophie, Religion, Kunst und Kultur in den römischen Oberschichten im 2. Jahrhundert bekanntermaßen en vogue war.

Die übrigen diskutierten Berührungspunkte zwischen Hadrian und

\footnotetext{
${ }^{57}$ Zur Adoption des L. Ceionius Commodus und den Folgen vgl. zuletzt Birley 1997, 289ff., zu (Cn. oder L. Pedanius) Fuscus (Salinator) PIR ${ }^{2}$ P 198; E. Groag, 'Pedanius', RE XIX (Stuttgart 1937), 19f.; Caballos Rufino 1990 I 2, $413 \mathrm{ff}$., Nr. I 44. Der genaue Ablauf der Ereignisse ist aufgrund der ungünstigen Quellenlage nur schwer zu rekonstruieren.

${ }^{58}$ Der Text auf einer Marmorbasis aus Lavinium (Ende des 1./Anfang des 2. Jahrhunderts) offenbart den Namen des dort anscheinend begüterten Senators L. Valerius Publicola Messala Helvidius Thrasea Priscus Minicius Natalis (AE 1998, 280). Bei diesem Mann könnte es sich um einen Enkel des jüngeren Minicius Natalis handeln, was bedeuten würde, daß dieser Nachkommen hatte. Eine andere Möglichkeit wäre, daß er die Namensbestandteile ,Minicius Natalis' durch Erbschaft erhielt.

${ }^{59}$ R. Syme, 'Antonine Government and Governing Class', Roman Papers V, 682.
} 
dem jüngeren Minicius Natalis unterstreichen diese Ansicht, zeigen sie doch einen Mann, der trotz seiner mehr oder weniger erfolgreichen Laufbahn und seiner guten Kontakte immer im Schatten anderer Senatoren und nicht zuletzt des Kaisers stand und in der letzten Regierungsphase Hadrians offenbar politisch „kaltgestellt" war, vielleicht aufgrund seiner oben vermuteten verwandtschaftlichen Beziehung zu den Pedanii. In den auf uns gekommenen literarischen Quellen hat er denn auch - Zufall oder nicht keinerlei Berücksichtigung erfahren. Dessen ungeachtet dürfte der Beitrag gezeigt haben, daß die folgenden Worte Edmund Groags heute mehr denn je ihre Gültigkeit besitzen: „Aus epigraphischem Material das Wesen einer Persönlichkeit $\mathrm{zu}$ erschließen, scheint wohl ein vergebliches Beginnen; immerhin lassen sich vielleicht gerade in unserem Falle [sc. L. Minicius Natalis Quadronius Verus] aus den Inschriften gewisse Schlüsse ziehen.“60

Düsseldorf, März 2003

${ }^{60}$ Groag 1932, 1840f. 


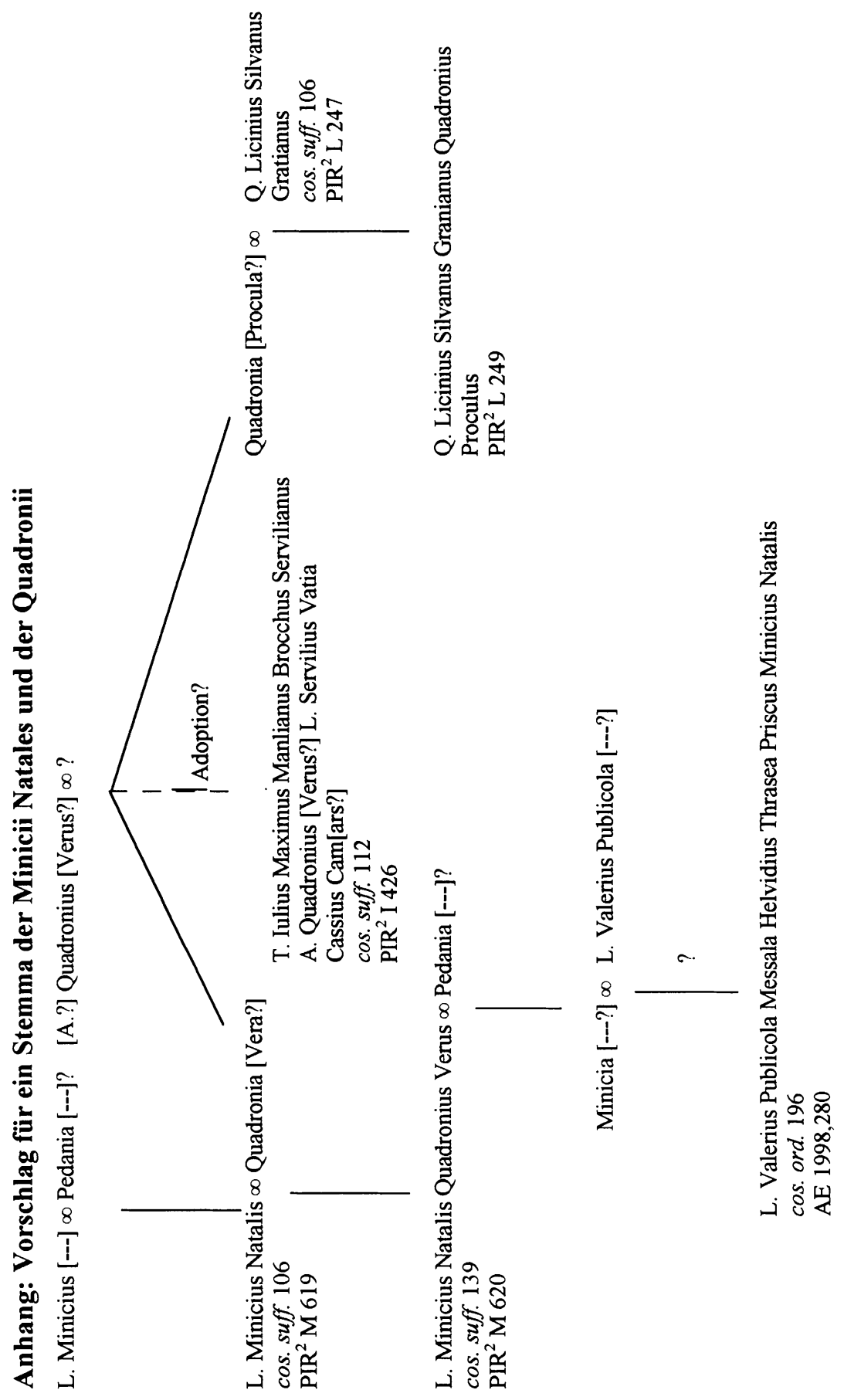

